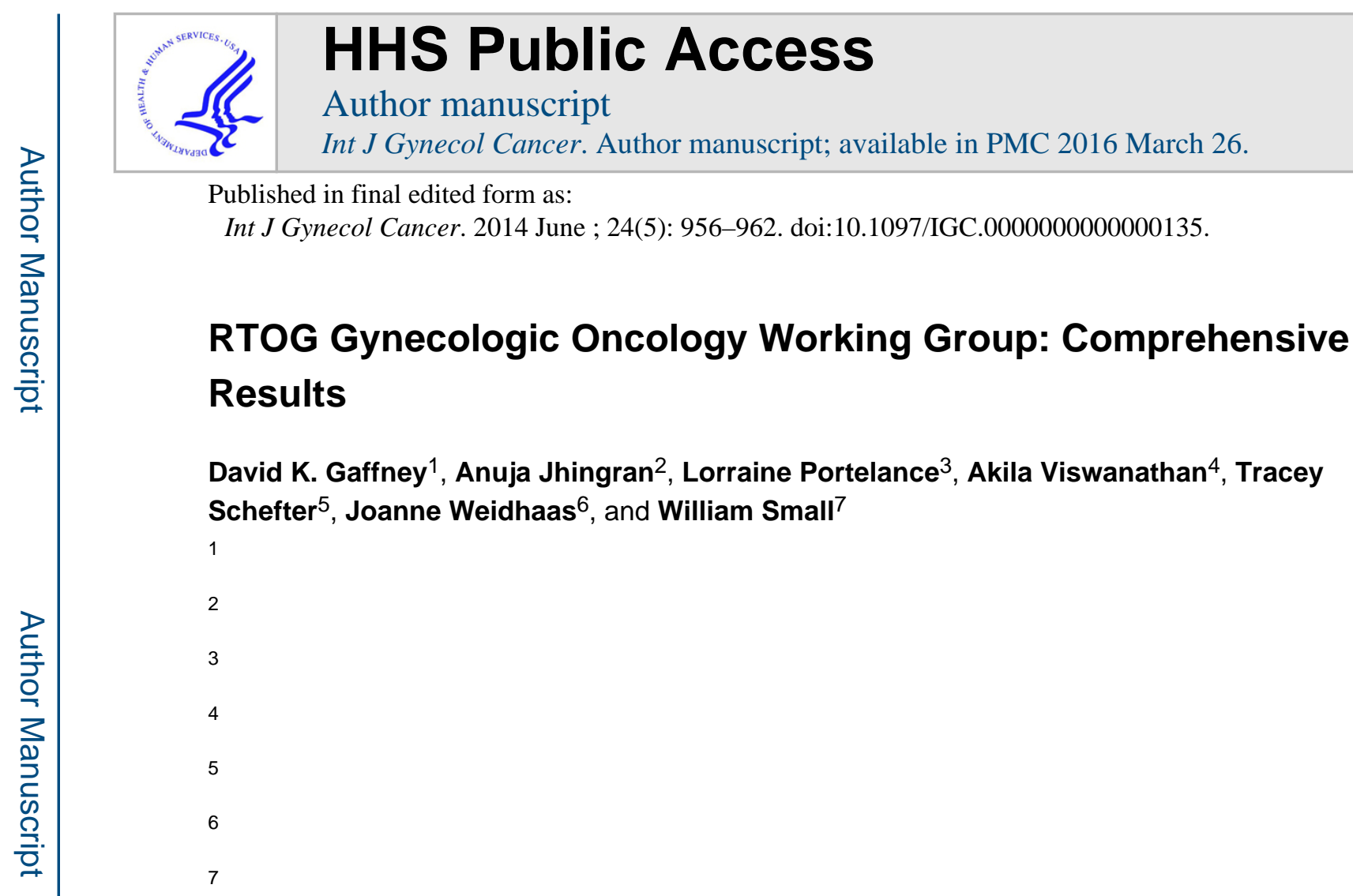

\title{
Abstract
}

The purpose of this report is to comprehensively describe the activities of the Gynecologic Oncology Working Group within the RTOG. Clinical trials will be reviewed as well as translational science and ancillary activities. Over the past 40 years, a myriad of clinical trials have been performed within the RTOG with the aim of improving overall survival and decreasing morbidity in women with cervical or endometrial cancer. Major study questions have included hyperbaric oxygen, neutron radiotherapy, altered fractionation, hypoxic cell sensitization, chemosensitization, and volume directed radiotherapy.

RTOG 7920 demonstrated improvement in overall survival in patients with stages IB through IIB cervical carcinoma receiving prophylactic paraaortic irradiation compared to pelvic radiation alone. RTOG 9001 demonstrated that cisplatin and 5-FU chemoradiotherapy to the pelvis for advanced cervix cancer markedly improved overall survival compared to extended field radiotherapy alone. More recent trials have employed radioprotectors, molecular targeted therapy, and intensity modulated radiation therapy. Ancillary studies have developed CTV atlases for research protocols and routine clinical use. Worldwide practice patterns have been investigated in cervix, endometrial, and vulvar cancer thru the Gynecologic Cancer Intergroup (GCIG).

Translational studies have focused on immunohistochemical markers, changes in gene expression, and miRNA patterns impacting prognosis.

The RTOG gynecologic working group has performed clinical trials that have defined the standard of care, improved survival, and added to our understanding of the biology of cervical and endometrial cancers.

Corresponding Author: David Gaffney MD PhD, Department of Radiation Oncology, University of Utah, Huntsman Cancer Hospital, 1950 Circle of Hope Rd., Salt Lake City, UT 84112, Tele 801581 2396, Fax 8015853502. 


\section{Early Clinical Trials}

RTOG gynecologic efforts are listed in chronologic order according to the following themes: early clinical trials, recent clinical trials, translational research and Gynecologic Cancer Intergroup efforts. In the late 1960's and early 1970's, there was substantial preclinical and clinical data available on the importance of hyperbaric oxygen, effects of fractionation, and utility of neutrons. Hyperbaric oxygen attempts to raise the partial pressure of oxygen in tissues to exploit the oxygen effect. Well oxygenated tissues are approximately 3 times more sensitive to radiation as anoxic tissues. Hyperfractionation theoretically may alter the therapeutic window by decreasing late effects while yielding similar tumoricidal effects. Neutrons are fundamentally different in that they are more heavily ionizing; that is, more destruction per track length. Consequently, the RTOG's first gynecologic trials were phase III questions addressing these issues. No benefit was identified with the experimental approach in these trials(table 1$)^{1-3}$. These early trials were underpowered to detect differences in the treatment arms: RTOG 7002 evaluating hyperbaric oxygen, RTOG 7105 testing standard fractionation versus split course, and RTOG 7608 comparing photons versus photons and neutrons had only 65, 287, and 156 patients each, respectively. These were ambitious efforts testing important, but technically challenging concepts such as hyperbaric oxygen and neutrons. Lessens in trial design were learned, and going forward greater statistical rigor has been adopted. Since the early 1980s, phase II efforts have been performed largely with the intent of advancing to phase III questions if an adequate signal is achieved.

Hypoxic cell sensitizers increase radiation induced free radical damage in hypoxic environments. After a phase I/II study was performed evaluating a nitroimidazole (misonidazole) as a radiation sensitizer, the RTOG embarked on a phase III study (80-05) in patients with stage IIIB and IVA cervix cancer (table 2) ${ }^{4,5}$. This trial randomized 120 patients between RT and RT + misonidazole from 1980 to 1984, and showed no improvement in pelvic control, disease free survival (DFS), overall survival (OS), or toxicity.

The RTOG has a strong history of evaluating optimal palliative regimens as well. RTOG 8502 was a phase III protocol for advanced pelvic malignancies. In the pilot portion 151 patients were accrued to a regimen receiving $44.4 \mathrm{~Gy}$ in 12 fractions ( $3.7 \mathrm{~Gy}$ bid) with a variable rest interval of 3-6 weeks after each 2 day consecutive course of $14.8 \mathrm{~Gy}$. The phase III portion randomized patients between a rest period of 2 or 4 weeks between fractions. There was no difference in late effects by length of rest period. Patients that received the full $44.4 \mathrm{~Gy}$ had a higher response rate. This fractionation scheme was found to produce good tumor regression, excellent palliation, and with a low incidence of complications ${ }^{6}$. This regimen is not widely used; however, it is effective and can be convenient for some patients.

The choice of the appropriate target volume has been a major theme in RTOG gynecologic studies. From 1979 thru 1986, RTOG 7920 randomized 367 patients to pelvic versus pelvic and paraaortic RT in patients with stage IB-IIB cervical cancer ${ }^{7}$. The $10 \mathrm{yr}$ OS rate was $44 \%$ in the pelvic arm and $55 \%$ in the extended field arm $(\mathrm{p}=0.02)$. The cumulative incidence of 
grade $4-5$ toxicities was doubled in the extended field arm ( $8 \%$ vs $4 \%, \mathrm{p}=0.06)$. In this trial, there was no requirement for surgical staging and cross sectional imaging was not used in all patients.

The RTOG has performed 2 trials evaluating twice daily RT in definitively treated cervix cancer patients: RTOG 8805 and RTOG 9210. The hypothesis was that higher biologic tumor doses can be delivered without increasing toxicity. RTOG 8805 was a phase II study in 81 patients delivering $1.2 \mathrm{~Gy}$ bid with a $4-6 \mathrm{hr}$ interfraction interval ${ }^{8}$. A grade 3 and 4 late toxicity rate at 3 years of $6 \%$ was felt to be promising. Hence, this concept was applied in RTOG 9210. Patients eligible had positive paraaortic lymph nodes and were treated with extended field together with chemotherapy consisting of cisplatin and 5-FU. Only $69 \%$ of patients completed RT per protocol and the grade 4 GI toxicity rate was $31 \%$. This regimen was felt to be too toxic without benefit compared to standard chemotherapy and radiotherapy (CRT).

RTOG participated in both Intergroup trials that changed standard of care in women treated by hysterectomy for cervix cancer. In GOG 92 (RTOG 8706) 277 stage I patients were randomized to 46-50.4 Gy RT to the pelvis versus observation after radical hysterectomy ${ }^{10}$. Patients were eligible if they had two or more of the following factors: lymph vascular space invasion (LVSI), $>1 / 3$ stromal invasion, or tumor $\geq 4 \mathrm{~cm}$. PFS was significantly increased with postoperative RT from 65\% to 78\% ${ }^{11}$. For SWOG 8797 (RTOG 9112, GOG 109) patients with high risk disease after radical hysterectomy defined as positive lymph nodes, positive parametria, or positive margins were randomized to RT alone or RT plus 4 cycles of cisplatin and 5-FU. OS was significantly improved by $10 \%$ with CRT with a concomitant $30 \%$ decrease in distant metastases, and $50 \%$ decrease in local failure ${ }^{12}$. In both these trials there was a particular benefit for patients with adenocarcinoma histology that received the experimental arm.

The addition of chemotherapy to radiotherapy in locally advanced disease was first found to be promising in RTOG 8515 , and hence, this was tested in a phase III design in RTOG $9001^{13}$. Patients with bulky stage IB through stage IVA disease were randomized to pelvic and paraaortic irradiation versus pelvic radiation plus 3 cycles of 5-FU and cisplatin chemotherapy. A $16 \%$ improvement in OS at 8 years (67\% versus $41 \%$ ) was achieved in the concomitant CRT arm ${ }^{14}$. The hazard ratio for the reduction in death with the addition of chemotherapy was 0.51 , and the rates of late complications were similar between the two arms. This landmark trial together with SWOG 8797 and 3 other GOG trials prompted an $\mathrm{NCI}$ alert in 1999 indicating a marked survival benefit for cisplatin-based RT in women with advanced cervix cancer, and promptly changed practice patterns worldwide.

\section{More recent clinical trials}

RTOG 9708 was RTOG's first trial performed exclusively in endometrial cancer. This phase II trial evaluated pelvic radiotherapy with concurrent cisplatin $50 \mathrm{mg} / \mathrm{m} 2$ on day 1 and day 28 followed by 4 cycles of extended adjuvant or outback carboplatin and paclitaxel chemotherapy given at 4 week intervals. Eligible patients had high risk stage I through stage IIIC1 disease. This trial was deemed to be safe and effective with a 4 year OS of $81 \%$ and 
no recurrences in patients with stage I-II disease ${ }^{15}$. This concept was advanced to a phase III trial; however, the trial had to be closed due to poor accrual (RTOG 9905). Currently, this CRT regimen is being tested in phase III trials in North America and Europe (GOG 0258 and PORTEC III, respectively). Additionally, this CRT regimen was used as the backbone for RTOG 0921. This phase II trial added Bevacizumab, an antibody directed against Vascular Endothelial Growth Factor (VEGF) given every 2 weeks for 3 times during the concomitant CRT for the same population as RTOG 9708. IMRT was used exclusively in this trial. RTOG 0921 completed accrual in December of 2011 after 34 patients were accrued $^{16}$. There were 2 local failures and 5 distant failures, and two year OS and DFS were $97 \%$ and $79 \%$, respectively.

The treatment of advanced cervix cancer with CRT can be morbid. In attempts to ameliorate treatment related morbidity RTOG 0116 evaluated amifostine as a radiation protector in a two part trial for patients with high common iliac or paraaortic metastases. In the initial phase, patients were treated with CRT alone and in part II amifostine was added. The acute grade 3-4 toxicity rates were $81 \%$ and $87 \%$, in parts I and II, respectively ${ }^{17}$. In RTOG 0128 , celecoxib was evaluated as a radiation sensitizer in 84 patients with stage IB-IVA pelvic confined cervical cancer and, again, no increased benefit was observed for the study agent in this phase II trial ${ }^{18}$. RTOG 0417 evaluated radiotherapy and cisplatin together with bevacizumab in the identical population to RTOG 0128. This was found to be safe and effective with $76 \%$ and $94 \%$ of patients receiving chemotherapy and RT per protocol, respectively ${ }^{19}$. The 3 year OS rate in RTOG 0417 was $80 \%$ and appears promising; however, LRF continues to be problematic.

After hysterectomy small bowel frequently fills the pelvis complicating RT. To ameliorate acute and late toxicity RTOG 0418 evaluated IMRT in the postoperative setting. Prior to the initiation of RTOG 0418, a CTV atlas was described and published on the web to allow appropriate target delineation on protocol ${ }^{20}$. Since this successful effort, many atlases have been developed throughout the RTOG for a variety of disease sites. Atlases used for CTV delineation are important tools in radiation oncology and this was one of the first developed, and currently is widely used. The primary endpoint in RTOG 0418 was to evaluate whether IMRT was transportable in a multi-institutional setting. This was felt achievable with $98 \%$ of cases having an acceptable IMRT plan ${ }^{21}$. It is of paramount importance to ensure that the local regional failure rate with IMRT does not exceed standard RT. Of 43 eligible women with endometrial cancer 4 patients had disease recurrence: regional in 2, suburethral in 1, and paraaortic in 1 for a local failure rate of $7 \%$. This translated to a 3 year OS rate of $92 \%$. Of 40 eligible patients with cervix cancer the 2 year rates for LRF, paraaortic failure, distant metastasis, and OS were $11 \%, 5 \%, 10 \%$, and $95 \%$, respectively. Thus, pelvic IMRT was found to be safe and effective and currently is used in a number of phase III trials.

Currently, the RTOG gynecologic oncology working group is participating in a number of phase III trials evaluating RT or CRT questions in endometrial or cervical cancer. RTOG 0724 is randomizing high risk cervix cancer patients after hysterectomy to standard CRT with weekly cisplatin versus the same plus 4 cycles of carboplatin and paclitaxel given in the extended adjuvant setting. The OUTBACK trial (RTOG 1174) has the exact same design for women with locally advanced intact cervix cancer (stages IB-IVA without positive para- 
aortic nodes). In GOG 0238 (RTOG 0973), pelvic RT is being compared with cisplatin plus standard RT for women with pelvic recurrences of endometrial cancer. In women with stage I and II endometrial cancer GOG 0249 (RTOG 1070) evaluated pelvic RT versus vaginal brachytherapy and 3 cycles of carboplatin and paclitaxel. GOG 0249 completed accrual and results are eagerly anticipated. For women with stage III and IVA endometrial cancer GOG 0258 (RTOG 1073) is randomizing patients to CRT as delivered in RTOG 9708 with concurrent cisplatin and 4 cycles of extended adjuvant chemotherapy versus 6 cycles of carboplatin and paclitaxel chemotherapy alone. GOG 0263 (RTOG 1171) is evaluating pelvic RT versus CRT employing weekly cisplatin for patients with intermediate risk features after hysterectomy.

\section{Translational Studies}

The RTOG Gynecologic Oncology Working Group has also been involved in a number of translational science studies (Table 3). A secondary analysis of RTOG 0116 and RTOG 0128 evaluated the importance of brachytherapy quality in 103 patients. It was found that asymmetry of tandem to ovoids, displacement of the ovoids in relation to the cervix, and poor packing adversely impacted local control ${ }^{22}$. One of the potential benefits for IMRT is a reduction in hematologic toxicity. This may be particularly important for delivery of concomitant CRT in either cervical or endometrial cancer. A secondary analysis from RTOG 0418 evaluated whether DVH parameters correlated with hematologic toxicity. In 83 patients bone marrow doses were determined and patients with a median bone marrow dose of $>34.2$ Gy had higher rates of grade $\geq 2$ hematologic toxicity ${ }^{23}$. Also in RTOG 0418, we evaluated whether oral contrast changed the dose delivered via treatment planning systems. There was found to be a small but statistically significant reduction in dose with the administration of oral contrast.

The RTOG currently is producing an atlas for the use in vulvar cancer to aid clinicians worldwide in target delineation in vulvar carcinoma. Additionally, the RTOG has rigorously evaluated inter-physician MRI contouring abilities in cervical cancer. Using kappa statistics, there was found to be a good agreement with the GTV for various pelvic organs ${ }^{24}$. Image guided brachytherapy with CT was compared with MRI. CT volumes were routinely larger; however, there was also slightly less variance between physicians with CT. CT was felt to be an acceptable replacement for MRI for image guided brachytherapy. The RTOG gynecologic cancer working group has also participated in standardizing pelvic organ contouring with the Genitourinary and Gastrointestinal Committees from the RTOG ${ }^{25}$.

Aneuploidy and percent S phase fraction from RTOG 9001 and the University of Miami were evaluated by flow cytometry on fresh tissue in 57 patients, and found to have no prognostic value ${ }^{26}$. RTOG 0128 had a number of scientific secondary endpoints. Tumor tissue biopsies were obtained prior to treatment and at the time of the first brachytherapy implant. RNA collection was feasible in a cooperative group study, and gene expression was found to correlate with clinical features such as histology, but not stage ${ }^{27,28}$. Additionally, using the differential gene expression signatures between the original biopsy and the biopsy at the initial implant, a 7 gene signature panel was identified that could predict outcome in advanced cervical cancer patients from $0128^{29}$. 
Immunohistochemistry (IHC) is a powerful technique for measuring protein expression and correlating clinical endpoints for well annotated samples in RTOG studies. In 51 patients from RTOG 0116 and 0128 , subunit expression of ribonucleotide reductase by IHC was prognostic in node positive patients ${ }^{30}$. From RTOG 0128, chromovision was compared with aqua technology for evaluating IHC. It was concluded that these technologies provided different results. Additionally, COX-2 was found to be inversely correlated with survival in this study ${ }^{31}$. A collaboration with Canadian investigators determined that ERCC1 expression is a negative prognostic factor in the advanced cervical cancer population ${ }^{32}$.

Fewer projects have been performed in endometrial cancer in the RTOG. However, one project used paraffin embedded tissues to evaluated microRNA expression as a prognostic factor, as well as the role of an inherited mutation in a microRNA binding site (the KRASvariant) as a genetic marker of risk. This study found that microRNAs may be predictive of certain clinical features, and that the KRAS-variant may be a genetic marker of risk of type II endometrial cancer. Follow on studies have evaluated microRNA signatures in cervical cancer tissues and results are being analyzed.

\section{Gynecologic Cancer Intergroup radiotherapy analyses}

The RTOG has also participated in secondary studies of the Gynecologic Cancer Intergroup (GCIG) (Table 4). The GCIG comprises over 20 cooperative groups worldwide. Four of these projects involved surveying members of these groups regarding practice patterns in cervix, endometrial, vulvar cancer, and the use of brachytherapy. The cervix cancer study identified that radiation dose and use of chemotherapy was similar worldwide, and hence, these should not be barriers to trial participation between groups ${ }^{33}$. The endometrial cancer survey demonstrated that the radiation dose was similar worldwide; however, there was no standardization of vaginal brachytherapy prescription ${ }^{34}$. In some countries it was customary to prescribe radiation to a set fraction of the vaginal length, and in others it was more common to prescribe to a precise length to the vaginal apex. In vulvar cancer, the radiation dose was similar worldwide; however, chemotherapy was not standardized ${ }^{35}$. A more recent survey evaluating practice patterns in brachytherapy demonstrated that cooperative groups in Asia used a lower dose compared to non-Asian cooperative groups ${ }^{36}$. For example, the external beam and brachytherapy equivalent doses for Asia and Europe were 71.2 Gy and 83.2 Gy, respectively. Other GCIG projects have included recommendations for staging and the importance of performing cervical cancer trials within the developing world where the greatest burden of disease exists 37,38 .

\section{Conclusions}

The RTOG Gynecologic Oncology Working Group has a rich history of performing informative phase II and phase III trials as well as ancillary studies. With the merger of NSABP, RTOG, and GOG to form NRG, the quality of gynecologic trials and the ancillary science will improve. Various expertise from the groups are complimentary. In the future, we will continue to evaluate the potential for personalized treatment. Advanced radiotherapeutic technologies such as IMRT, IGRT, and IGBT will be rigorously tested in defined patient subsets to discern if they improve the therapeutic window for gynecologic 
patients. Imaging biomarkers such as MRI or PET modalities may be important as early response indicators permitting dose escalation or de-escalation in advanced cervix cancer. We will also continue to evaluate protein expression, mRNA and micro-RNA gene signatures and genomic technologies to potentially exploit chemotherapy or radiation sensitivity. It will be important to continue to refine appropriate treatment populations to achieve better overall survival, a reduction in morbidity, an improvement in quality of life, and to promote cost effectiveness.

\section{References}

1. Brady LW, Plenk HP, Hanley JA, et al. Hyperbaric oxygen therapy for carcinoma of the cervixstages IIB, IIIA, IIIB and IVA: results of a randomized study by the Radiation Therapy Oncology Group. Int J Radiat Oncol Biol Phys. 1981 Aug; 7(8):991-998. [PubMed: 7028700]

2. Marcial VA, Amato DA, Marks RD, et al. Split-course versus continuous pelvis irradiation in carcinoma of the uterine cervix: a prospective randomized clinical trial of the Radiation Therapy Oncology Group. Int J Radiat Oncol Biol Phys. 1983 Apr; 9(4):431-436. [PubMed: 6406397]

3. Maor MH, Gillespie BW, Peters LJ, et al. Neutron therapy in cervical cancer: results of a phase III RTOG Study. Int J Radiat Oncol Biol Phys. 1988 May; 14(5):885-891. [PubMed: 3283085]

4. Meoz RT, Spanos WJ, Doss L, et al. Misonidazole combined with large-fraction pelvic irradiation in the treatment of patients with advanced pelvic malignancies. Preliminary report of an ongoing RTOG phase I-II study. Am J Clin Oncol. 1983 Aug; 6(4):417-422. [PubMed: 6191560]

5. Grigsby PW, Winter K, Wasserman TH, et al. Irradiation with or without misonidazole for patients with stages IIIB and IVA carcinoma of the cervix: final results of RTOG 80-05. Radiation Therapy Oncology Group. Int J Radiat Oncol Biol Phys. 1999 Jun 1; 44(3):513-517. [PubMed: 10348279]

6. Spanos WJ Jr, Perez CA, Marcus S, et al. Effect of rest interval on tumor and normal tissue response-a report of phase III study of accelerated split course palliative radiation for advanced pelvic malignancies (RTOG-8502). Int J Radiat Oncol Biol Phys. 1993 Feb 15; 25(3):399-403. [PubMed: 7679668]

7. Rotman M, Pajak TF, Choi K, et al. Prophylactic extended-field irradiation of para-aortic lymph nodes in stages IIB and bulky IB and IIA cervical carcinomas. Ten-year treatment results of RTOG 79-20. JAMA. 1995 Aug 2; 274(5):387-393. [PubMed: 7616634]

8. Komaki R, Pajak TF, Marcial VA, et al. Twice-daily fractionation of external irradiation with brachytherapy in bulky carcinoma of the cervix. Phase I/II study of the Radiation Therapy Oncology Group 88-05. Cancer. 1994 May 15; 73(10):2619-2625. [PubMed: 8174061]

9. Grigsby PW, Lu JD, Mutch DG, et al. Twice-daily fractionation of external irradiation with brachytherapy and chemotherapy in carcinoma of the cervix with positive para-aortic lymph nodes: Phase II study of the Radiation Therapy Oncology Group 92-10. Int J Radiat Oncol Biol Phys. 1998 Jul 1; 41(4):817-822. [PubMed: 9652843]

10. Sedlis A, Bundy BN, Rotman MZ, et al. A randomized trial of pelvic radiation therapy versus no further therapy in selected patients with stage IB carcinoma of the cervix after radical hysterectomy and pelvic lymphadenectomy: A Gynecologic Oncology Group Study. Gynecol Oncol. 1999 May; 73(2):177-183. [PubMed: 10329031]

11. Rotman M, Sedlis A, Piedmonte MR, et al. A phase III randomized trial of postoperative pelvic irradiation in Stage IB cervical carcinoma with poor prognostic features: follow-up of a gynecologic oncology group study. Int J Radiat Oncol Biol Phys. 2006 May 1; 65(1):169-176. [PubMed: 16427212]

12. Peters WA 3rd, Liu PY, Barrett RJ 2nd, et al. Concurrent chemotherapy and pelvic radiation therapy compared with pelvic radiation therapy alone as adjuvant therapy after radical surgery in high-risk early-stage cancer of the cervix. J Clin Oncol. 2000 Apr; 18(8):1606-1613. [PubMed: 10764420]

13. John M, Flam M, Caplan R, et al. Final results of a phase II chemoradiation protocol for locally advanced cervical cancer: RTOG 85-15. Gynecol Oncol. 1996 May; 61(2):221-226. [PubMed: 8626137] 
14. Eifel PJ, Winter K, Morris M, et al. Pelvic irradiation with concurrent chemotherapy versus pelvic and para-aortic irradiation for high-risk cervical cancer: an update of radiation therapy oncology group trial (RTOG) 90-01. J Clin Oncol. 2004 Mar 1; 22(5):872-880. [PubMed: 14990643]

15. Greven K, Winter K, Underhill K, et al. Final analysis of RTOG 9708: adjuvant postoperative irradiation combined with cisplatin/paclitaxel chemotherapy following surgery for patients with high-risk endometrial cancer. Gynecol Oncol. 2006 Oct; 103(1):155-159. [PubMed: 16545437]

16. Viswanathan AN, Moughan J, Miller BE, et al. A phase 2 study of postoperative intensity modulated radiation therapy with concurrent cisplatin and bevacizumab followed by carboplatin and paclitaxel for patients with endometrial cancer: one year results of RTOG 0921. Int J Radiat Oncol Biol Phys. 2013 Oct 1.87(25):S4.

17. Small W Jr, Winter K, Levenback C, et al. Extended-field irradiation and intracavitary brachytherapy combined with cisplatin and amifostine for cervical cancer with positive para-aortic or high common iliac lymph nodes: results of arm II of Radiation Therapy Oncology Group (RTOG) 0116. Int J Gynecol Cancer. 2011 Oct; 21(7):1266-1275. [PubMed: 21892091]

18. Gaffney DK, Winter K, Dicker AP, et al. Efficacy and patterns of failure for locally advanced cancer of the cervix treated with celebrex (celecoxib) and chemoradiotherapy in RTOG 0128. Int J Radiat Oncol Biol Phys. 2007 Sep 1; 69(1):111-117. [PubMed: 17482376]

19. Schefter TE, Winter K, Kwon JS, et al. A Phase II Study of Bevacizumab in Combination with Definitive Radiotherapy and Cisplatin Chemotherapy in Untreated Patients with Locally Advanced Cervical Carcinoma: Preliminary Results of RTOG 0417. Int J Radiat Oncol Biol Phys. 2012 Jul 15; 83(4):1179-84. [PubMed: 22342094]

20. Small W Jr, Mell LK, Anderson P, et al. Consensus guidelines for delineation of clinical target volume for intensity-modulated pelvic radiotherapy in postoperative treatment of endometrial and cervical cancer. Int J Radiat Oncol Biol Phys. 2008 Jun 1; 71(2):428-434. [PubMed: 18037584]

21. Jhingran A, Winter K, Portelance L, et al. A Phase II Study of Intensity Modulated Radiation Therapy to the Pelvis for Postoperative Patients With Endometrial Carcinoma: Radiation Therapy Oncology Group Trial 0418. Int J Radiat Oncol Biol Phys. 2012 Sep 1; 84(1):e23-8. [PubMed: 22543211]

22. Viswanathan AN, Moughan J, Small W Jr, et al. The quality of cervical cancer brachytherapy implantation and the impact on local recurrence and disease-free survival in radiation therapy oncology group prospective trials 0116 and 0128. Int J Gynecol Cancer. 2012 Jan; 22(1):123-131. [PubMed: 22193645]

23. Klopp AH, Moughan J, Portelance L, et al. Hematologic toxicity in RTOG 0418: a phase 2 study of postoperative IMRT for gynecologic cancer. Int J Radiat Oncol Biol Phys. 2013 May 1; 86(1): 83-90. [PubMed: 23582248]

24. Lim K, Small W Jr, Portelance L, et al. Consensus guidelines for delineation of clinical target volume for intensity-modulated pelvic radiotherapy for the definitive treatment of cervix cancer. Int J Radiat Oncol Biol Phys. 2011 Feb 1; 79(2):348-355. [PubMed: 20472347]

25. Gay HA, Barthold HJ, O’Meara E, et al. Pelvic Normal Tissue Contouring Guidelines for Radiation Therapy: A Radiation Therapy Oncology Group Consensus Panel Atlas. Int J Radiat Oncol Biol Phys. 2012 Jul 1; 83(3):e353-62. [PubMed: 22483697]

26. Wolfson AH, Winter K, Crook W, et al. Are increased tumor aneuploidy and heightened cell proliferation along with heterogeneity associated with patient outcome for carcinomas of the uterine cervix? A combined analysis of subjects treated in RTOG 9001 and a single-institution trial. Int J Radiat Oncol Biol Phys. 2008 Jan 1; 70(1):111-117. [PubMed: 17919835]

27. Gaffney DK, Winter K, Fuhrman C, et al. Feasibility of RNA collection for micro-array gene expression analysis in the treatment of cervical carcinoma: a scientific correlate of RTOG C-0128. Gynecol Oncol. 2005 May; 97(2):607-611. [PubMed: 15863167]

28. Zempolich K, Fuhrman C, Milash B, et al. Changes in gene expression induced by chemoradiation in advanced cervical carcinoma: a microarray study of RTOG C-0128. Gynecol Oncol. 2008 May; 109(2):275-279. [PubMed: 18299147]

29. Weidhaas JB, Li SX, Winter K, et al. Changes in gene expression predicting local control in cervical cancer: results from Radiation Therapy Oncology Group 0128. Clin Cancer Res. 2009 Jun 15; 15(12):4199-4206. [PubMed: 19509178] 
30. Kunos CA, Winter K, Dicker AP, et al. Ribonucleotide reductase expression in cervical cancer: a radiation therapy oncology group translational science analysis. Int J Gynecol Cancer. 2013 May; 23(4):615-621. [PubMed: 23552804]

31. Doll CM, Winter K, Gaffney DK, et al. COX-2 expression and survival in patients with locally advanced cervical cancer treated with chemoradiotherapy and celecoxib: a quantitative immunohistochemical analysis of RTOG C0128. Int J Gynecol Cancer. 2013 Jan; 23(1):176-183. [PubMed: 23221733]

32. Doll CM, Aquino-Parsons C, Pintilie M, et al. The significance of tumoral ERCC1 status in patients with locally advanced cervical cancer treated with chemoradiation therapy: a multicenter clinicopathologic analysis. Int J Radiat Oncol Biol Phys. 2013 Mar 1; 85(3):721-727. [PubMed: 22836058]

33. Gaffney DK, Du Bois A, Narayan K, et al. Practice patterns of radiotherapy in cervical cancer among member groups of the Gynecologic Cancer Intergroup (GCIG). Int J Radiat Oncol Biol Phys. 2007 Jun 1; 68(2):485-490. [PubMed: 17336465]

34. Small W Jr, Du Bois A, Bhatnagar S, et al. Practice patterns of radiotherapy in endometrial cancer among member groups of the gynecologic cancer intergroup. Int J Gynecol Cancer. 2009 Apr; 19(3):395-399. [PubMed: 19407566]

35. Gaffney DK, Du Bois A, Narayan K, et al. Patterns of care for radiotherapy in vulvar cancer: a Gynecologic Cancer Intergroup study. Int J Gynecol Cancer. 2009 Jan; 19(1):163-167. [PubMed: 19258960]

36. Viswanathan AN, Creutzberg CL, Craighead P, et al. International brachytherapy practice patterns: a survey of the Gynecologic Cancer Intergroup (GCIG). Int J Radiat Oncol Biol Phys. 2012 Jan 1; 82(1):250-255. [PubMed: 21183288]

37. Petru E, Luck HJ, Stuart G, et al. Gynecologic Cancer Intergroup (GCIG) proposals for changes of the current FIGO staging system. Eur J Obstet Gynecol Reprod Biol. 2009 Apr; 143(2):69-74. [PubMed: 19195765]

38. Kitchener HC, Hoskins W, Small W Jr, et al. The development of priority cervical cancer trials: a Gynecologic Cancer InterGroup report. Int J Gynecol Cancer. 2010 Aug; 20(6):1092-1100.

[PubMed: 20683424] 


\section{Table 1}

RTOG phase III gynecologic clinical trials

\begin{tabular}{|c|c|c|c|}
\hline Study \# & Years & Study Design & Result \\
\hline 7002 & $1972-1975$ & RT vs. RT + hyperbaric oxygen & Survival same \\
\hline 7105 & $1965-1980$ & Standard fractionation vs. split-course & Survival same \\
\hline 7608 & $1976-1984$ & Photons vs. photons + neutrons (external) & Survival same \\
\hline 7920 & $1979-1986$ & Pelvic RT vs. Pelvic + Paraaortic RT & Improved survival for paraaortic RT \\
\hline 8005 & $1980-1984$ & RT vs. $\mathrm{RT}+$ misonidazole & Survival same \\
\hline 8502 & 1985-1989 & Palliative RT (short vs long rest interval) & Similar \\
\hline 8514 & $1977-1980$ & Photons vs. photons + neutrons (Cf-252) & Terminated due to poor accrual \\
\hline $8706^{*}$ & $1988-1989$ & RT vs Observation after hysterectomy & Improved PFS with RT \\
\hline 9001 & $1990-1997$ & RT vs. chemoradiotherapy (CRT) & Improved survival for RT + chemo \\
\hline $9112^{*}$ & 1991-1996 & RT vs CRT for post op cervix & Improved survival for CRT \\
\hline $9905^{*}$ & $2000-2003$ & RT vs CRT + carbo/taxol & Closed for poor accrual \\
\hline $0724^{*}$ & 2009 & CRT vs CRT + carbo/taxol & On going \\
\hline $0238^{* \wedge}$ & $2007-$ & RT vs CRT for rec. uterine ca. & On going \\
\hline $0249^{* \wedge}$ & $2008-$ & Pelvic RT vs VCB in stage I/II uterine ca. & On going \\
\hline $0258^{* \wedge}$ & $2009-$ & CRT vs Chemo in stage III/IV uterine ca. & On going \\
\hline $0263^{* \wedge}$ & $2010-$ & RT vs CRT post op intermed. risk cervix & On going \\
\hline $1174^{*}$ & 2012 & CRT vs CRT + carbo/taxol & On going \\
\hline
\end{tabular}

Abbreviations: RT radiotherapy, CRT chemoradiotherapy, PFS progression free survival, VCB vaginal cuff Brachytherapy, Carbo carboplatin, op operative 
Table 2

RTOG phase II gynecologic clinical trials

\begin{tabular}{llll}
\hline Study \# & Years & Study Design & Result \\
\hline 7905 & $1979-1982$ & 10 Gy $\times$ 3 + misonidazole & Too toxic \\
8515 & $1985-1988$ & Chemo + RT in adv. cervix & Promising \\
8805 & $1988-1989$ & Bid RT in adv. cervix & Safe and effective \\
9210 & $1992-1995$ & Bid CRT in adv. cervix & Too toxic \\
9708 & $1997-1999$ & CRT + outback carbo/taxol in endo & Safe and effective \\
0116 & $2001-2007$ & CRT + amifostine in PA node + cervix & No benefit for amifostine \\
0128 & $2001-2004$ & CRT + Celebrex in adv. cervix & No benefit for Celebrex \\
0417 & $2006-2009$ & CRT + Bevacizumab in adv. cervix & Safe and effective \\
0418 & $2006-2008$ & IMRT for post op cervix/endo & Transportable, safe, effective \\
0921 & $2009-2011$ & IMRT/Bevacizumab + outback carbo/taxol & Safe \\
\hline
\end{tabular}


Table 3

RTOG translation science from gynecologic working group

\begin{tabular}{lll}
\hline Study \# & Hypothesis/Question & Result \\
\hline 9001 & Does aneuploidy or S phase fraction predict outcome? & No \\
$0116 / 0128$ & Is brachytherapy quality important? & Asymmetry, displacement and poor packing decreased outcome \\
0128 & Is RNA collection feasible in a cooperative group study? & Yes \\
0128 & Does gene expression correlate with clinical features? & Gene expression correlates with histology \\
0128 & Does a gene signature predict outcome? (Weidhaas) & 7 gene signature panel predicts outcome \\
0418 & What is the appropriate target post hysterectomy? (Small) & CTV Template generated for international use \\
0418 & Do DVH parameters correlate with Heme toxicity? (AK) & Decreased Heme toxicity with less dose to bone marrow \\
0418 & Does contrast change dose? & Bowel contrast reduces dose to target \\
$0116 / 0128$ & Ribonuclotide reductase as a prognostic factor? & Reduce OS in cohorts with increased expression by IHC \\
$9905 / 9708$ & Does the KRAS-variant miRNA predict outcome? & Yes \\
0128 & Chromavision vs AQUA for CD34 and COX2? & COX2 inversely correlated with survival \\
0128 & P53/ERCC1/Ki67 as prognostic factors? & ERCC1 is an adverse prognostic factor \\
Other & Pratice patterns in vulvar cancer? (King) & Low utilization rate of IMRT \\
Other & Consensus guidelines in intact cervix? (lim) & GTV agreement high with MRI \\
Other & Normal tissue contouring? (Gay) & Pelvic organ contouring standardized \\
Other & CT vs MR for image guided brachytherapy? & Both CT and MR are acceptable \\
\hline
\end{tabular}


Table 4

RTOG participation in secondary studies of the Gynecologic Cancer Intergroup

\begin{tabular}{lll}
\hline Year & Hypothesis/Question & Results \\
\hline 2007 & Global practice patterns in cervix cancer & RT dose and use of chemo similar worldwide \\
2009 & Global practice patterns in endometrial cancer & RT dose similar. Vaginal brachytherapy not standarized \\
2009 & Global practice patterns in vulvar cancer & RT dose similar, use of chemo not standardized \\
2009 & Proposal for changes in Staging of Gyn cancers & Multiple changes suggested \\
2010 & Priority of cervix cancer trials & Need for trials in developing world \\
2012 & Global practice patterns in brachytherapy & Asia uses a lower dose \\
\hline
\end{tabular}

